\title{
La modification adjectivale en anaphore associative : le cas de
}

\section{l'église romane}

\section{Mathilde Salles}

\begin{abstract}
Mathilde Salles : Associative anaphora and adjectival modification

Adding a modifier in the anaphoric NP can impede the good formation of the associative anaphora. If, in the famous example ofthe village and the church (Un village... L"église...), we substitute l"église romane for l"église, the anaphoric séquence becomes déviant. According to G. Kleiber, this kind of séquence is not well-formed because there is no stereotypical relation between the two référents. We can find however well-formed associative anaphors with a non stereotypical adjectival modifier in the anaphoric NP. In this case, two factors, which are linked to the value of existential uniqueness that the definite article présupposes, are décisive : $1^{\circ}$ the restrictive or descriptive feature ofthe adjective ; $2^{\circ}$ if the adjective has a restrictive feature, the stereotypical amount of " parts".
\end{abstract}

\section{Citer ce document / Cite this document :}

Salles Mathilde. La modification adjectivale en anaphore associative : le cas de l'église romane. In: Langages, 40e année, $\mathrm{n}^{\circ} 163$. 2006. Unité(s) du texte. pp. 25-36.

doi : 10.3406/lgge.2006.2681

http://www.persee.fr/doc/lgge_0458-726x_2006_num_40_163_2681

Document généré le 21/10/2015 


\section{La modification adjectivale en anaphore associative : le cas de l'église romane}

L'unité du texte a partie liée avec les marques de cohésion, même si celles-ci, on le sait, ne suffisent nullement à garantir son unité globale, sa cohérence. Qu'il soit extrêmement facile de construire des « non-textes » fortement colnésifs et qu'il ne soit pas impossible de trouver des textes (sans doute toujours très courts) sans marques explicites de cohésion ( cf. les exemples proposés par G. Brown et G. Yule 1983, 196) ne signifie évidemment pas que les liens cohésifs, éléments qui relient, joignent, rassemblent, qui unissent, ne jouent aucun rôle dans la constitution du texte comme totalité organisée. Le lien cohésif auquel on va s'attacher ici est un lien anaphorique qui a ceci d'original par rapport à la plupart des anaphores qu'il combine cohésion-répétition et progression, continuité et nouveauté (cf. G. Kleiber 2001, 13-14). En mêlant ces deux aspects complémentaires de la répartition de l'information, l'anaphore associative, puisqu'il s'agit d'elle, contribue ainsi doublement à l'unité du texte.

Dans une série de travaux destinés à montrer que l'anaphore associative (désormais AA) « roule bien sur des stéréotypes " ${ }^{1}$, Kleiber (1993a et b, 1994a et $b, 2001)$ souligne que l'ajout d'un modificateur restrictif dans le SN anaphorique est susceptible d'entraver le bon fonctionnement de la relation anaphorique. $\mathrm{Si}$, dans l'exemple désormais célèbre du village et de l'église, on substitue l'église romane à l'église, la séquence anaphorique devient difficile:

(1) a. Nous entrâmes dans un village. L'église était située sur une hauteur. (Kleiber 1993a, 361 et 1993b, 57)

b. ? Nous entrâmes dans un village. L'église romane était située sur une hauteur. (Kleiber 1993a, 361 et 1993b, 57)

On observe le même phénomène dans la paire d'exemples suivante :

(2) a. J'ai jeté un coup d'ail dans une pièce. Le plafond était très haut. (d'après Clark 1977, 415)

b. ? J'ai jeté un coup d'ail dans une pièce. Le plafond jaune étnit très haut. (Kleiber 1993b, 57)

1. La formule est de Kleiber. 
Dans l'analyse de Kleiber, ces mauvais enchaînements constituent un argument supplémentaire en faveur de la thèse lexico-stéréotypique de l'AA. Les séquences ( $1 \mathrm{~b}$ ) et (2b) seraient mal formées à cause du manque de relation conventionnelle entre les deux types de référents : à un village, le savoir conventionnel associe une église, mais pas, de façon plus spécifique, une église romane ; parmi les parties stéréotypiques d'une pièce, on reconnaît un plafond, mais pas un plafond jaune.

\section{OBJECTIONS À UNE EXPLICATION EN TERMES STRICTEMENT STÉRÉOTYPIQUES}

Cette explication soulève toutefois deux objections.

\subsection{Contre-exemples}

Tout d'abord, elle se heurte à des contre-exemples manifestes :

(3) "Au dernier crépuscule, aux premières étoiles, ces dames arrivent, avec des révérences adorables. Et bientôt, notre maisonnette est pleine de petites femmes accronpies, dont les yeux bridés sourient vaguement, on voit lwire comme de l'ébène poli tous les beaux chignons aux coques soignées; les corps frêles se perdent dans les plis des vêtements trop larges, qui bâillent tous, comme prêts à tomber, sur les petits dos fuyants, et découvent les nuques exquises, " $(P$. Loti, Madame Chrysanthème, Calmann-Lévy, 1925, p. 275-6)

(4) J'ai jeté un coup d'ail dans une pièce. Le plafond jaune jurait avec les murs rouges.

(5) "Nous habitions un grand appartement plein de choses étranges. (...) Tout l'appartement était rempli de curiosités naturelles. Seul, le petit salon n'avait été envalui ni par la zoologie, ni par la minéralogie, ni par l'ethnographie, ni par la tératologie (...) » (A. France, Le livre de mon ami, CEuvres, tome I, La Pléiade, Gallimard, 1984, p. 445)

Aucune de ces séquences n'est déviante bien que le savoir conventionnel n'associe ni des petits dos fuyants ou des nuques exquises aux femmes japonaises, ni un plafond jaune à une pièce, ni - je crois - un petit salon à un appartement, même grand. Toutes ces spécifications sont contingentes. Cela dit, ces contre-exemples n'ont peut-être pas tous la même validité ; certains d'entre eux ne présentent vraisemblablement pas des modificateurs à valeur restrictive. On reviendra sur ce point plus fard.

\subsection{Le cas des optionnels}

Enfin, problème sans doute plus important, l'explication en terme d'absence de savoir conventionnel avancée par Kleiber paraît entrer en contradiction avec celle proposée pour expliquer l'occurrence possible de composants ${ }^{2}$ optionnels

2. Le terme est volontairement assez vague de façon à inclure toutes les relations sémantiques pertinentes en AA (sur ces diverses relations sémantiques, $c f$. la typologie des AA établie par Kleiber 2001). On emploiera aussi quelquefois le terme de partie dans un sens aussi large que celui de composant. 
en AA. La question des optionnels (les "inducible parts » et les " optional roles » de H. Clark 1977), illustrée par les exemples (6) et (7) :

(6) Jean a été assassiné hier. Le couteau a été retrouvé à proximité. (Clark 1977, 417, repris par Kleiber 1993a, 365)

(7) Je suis entré dans une pièce. Les chandeliers brillaient vivenent. (Kleiber 1993a, 365, à partir de Clark 1977, 416)

aurait pu constituer un obstacle majeur à la thèse lexico-stéréotypique, puisque ces éléments optionnels ne sont, par définition, ni nécessaires, ni typiques. En effet, le savoir conventionnel associe à un assassinat une arme et à une pièce des éléments de mobilier, mais pas de façon plus spécifique un couteau et des chandeliers. Doiton, pour autant, conclure à une totale absence de lien stéréotypique entre les entités mises en jeu dans les exemples (6) et (7) ? La réponse de Kleiber est négative. L'association stéréotypique est sauvegardée, dans la mesure où ces composants définissent chacun un composant possible $a$ priori de l'élément inclusif : dans les exemples (6) et (7), couteau et chandeliers constituent respectivement une arme possible a priori pour un meurtre et un élément de mobilier possible a priori pour une pièce. Aucun ne définit un élément marginal et, par conséquent, aucun ne rend le « tout ${ }^{3}$ atypique. Et Kleiber $(1993 a, 367)$ conclut en ces termes :

«La non-application de la règle d'inférence si $x$ est $Y$, alors $x$ a généralement/normalement $W^{4}$ n'est ainsi pas suffisante pour conclure à l'absence d'un lien associatif stéréotypique. L'ingrédient n'a pas besoin d'être majoritairement présent pour donner lieu à une règle par défaut d'origine probabiliste. Il suffit que sa présence dans le tout soit possible a priori. La stéréotypie est par là même sauve. "

Seulement, si la contrainte est telle que l'énonce Kleiber (1993a, 366) : « 11 faut que la "partie" soit telle qu'elle ne rende pas atypique le tout ", on ne comprend plus pourquoi l'église romane et le plafond jaune produisent des séquences anaphoriques mal formées en (1b) et (2b) et surtout pourquoi le statut de composants possibles leur est refusé (rappelons que, selon Kleiber, il n'y a plus ici de relation conventionnelle entre les deux types de référents). L'église romane et le plafond jaune des exemples (1b) et (2b) ne constituent pas plus des éléments marginaux pour un village et une pièce que ne le sont le couteau et les chandeliers pour un assassinat et une pièce. Ou encore, pour dire les choses autrement, un village avec une église romane n'a rien d'atypique, la présence d'une église romane dans un village semble bien possible a priori. La spécification adjectivale romane concourt simplement à faire de l'élément typique église un élément possible (église romane).

Du point de vue de la stéréotypie, le cas de l'église romane (ou du plafond jaune) ne paraît pas différent de celui des optionnels de Kleiber. Le double test en mais ${ }^{5}$, utilisé par Kleiber (1993b, 1995 et 2001) comme révélateur de la stéréotypie

3. Au sens large du terme.

4. Règle à laquelle répondent les composants typiques; les composants possibles, eux, répondront à une règle du type si $x$ est $Y$, alors $x$ peut avoir $W$.

5. Double test qui utilise des séquences de la forme $p$ mais $q$ et $p$ mais non q, dans lesquelles, «la proposition $p$ est une structure d'appartenance catégorielle du type C'est un $N$ où $\mathrm{N}$ représente le substantif dont on veut mettre en relief le contenu sémantique, alors que la proposition $q$ forme le trait soumis à examen. " (Kleiber 1995, 77-78). 
lexicale, confirme d'ailleurs cette parenté. D. Cruse (1986) utilise ce test afin de ditférencier traits attendus (ou encore typiques, probables), traits inattendus et traits possibles. Les traits attendus acceptent, de façon normale, la version négative $p$ mais non $q(c f .(8)$ ), mais refusent la version positive $p$ mais $q$ (cf. (9)) :

(8) a. C'est une voiture, mais elle n'a pas de moteur.

b. C'est un village, mais il n'y a pas d'église.

(9) a. ? C'est une voiture, mais elle a un moteur.

b. ? C'est un village, mais il y a une église.

La bizarrerie provoquée par la version positive confirme que les traits « avoir un moteur ", " comprendre une église " font bien partie des propriétés qu'on attend pour une voiture et un village.

Les traits inattendus ont, vis-à-vis de la normalité des plurases-tests en mais, un comportement exactement inverse à celui des traits attendus; leur occurrence dans la version positive produit une plurase normale, décrivant un membre inhabituel, peu ordinaire :

(10) a. C'est un chien, mais il peut chanter. (exemple que Cruse 1986, 17, commente en ces termes : "phrase normale, chien inhabituel ")

b. C'est un village, mais il y a un grand magasin. (pour reprendre un couple, inapte à l'AA6 ${ }^{6}$, proposé par Kleiber 1993a et b)

Leur occurrence dans la version négative est inappropriée:

(11) a. ? C'est un chien, mais il ne peut pas chanter.

b. ? C'est un villnge, mais il n'y a pas de grand magasin.

Enfin, les traits possibles produiront des plurases bizarres dans les deux cas:

(12) a. ? C'est un chien, mais il est marron. ("Pourquoi ne le serait-il pas?" s'interroge Cruse 1986, 17)

b. ? C'est une pièce, mais il y a des chandeliers. (Pourquoi n'y en aurait-il pas ?)

c. ? C'est un assassinat, mais il a été commis avec un contenu. (Pourquoi n'aurait-il pas été commis avec un couteau ?)

(13) a. ? C'est un chien, mais il n'est pas marron. (" Pourquoi le serait-il ? " s'interroge Cruse 1986, 17)

b. ? C'est wne pièce, mais il n'y a pas de chandeliers. (Pourquoi y en aurait-il ?)

c. ? C'est un assassinat, mais il n'a pas été commis avec un couteau. (Pourquoi aurait-il été commis avec un couteau ?)

Vis-à-vis de ces tests en mais, l'église romane et le plafond jaune des exemples (1b) et (2b) se comportent comme des traits possibles, et non comme des éléments - tels les traits inattendus de Cruse - qui n'entretiendraient aucun lien conventionnel avec village et pièce. Leur occurrence est inappropriée dans les deux versions et elle suscite exactement le même type d'interrogations:

(14) a. ? C'est un village, mais il y a une église romane. (Pourquoi n'y aurait-il pas d'église romane ?)

b. ? C'est une pièce, mais ellc a un plafond jaune. (Pourquoi ne serait-il pas jaune?)

6. Inapte à l'AA à cause du défaut de relation stéréotypique. 
a. ? C'est un village, mais il n'y a pas d'église romane. (Pourquoi y aurait-il une église romane?)

b. ? C'est une pièce, mais elle n'a pas de plafond jaume. (Pourquoi serait-iI jaune ?)

S'il y a encore du savoir conventionnel à l'œuvre entre pièce et chandelier, assassinat et couteau, il semble bien qu'il y en ait aussi entre village et église romane, pièce et plafond jaune. Mais alors, pourquoi, si une église romane est bien un composant possible d'un village, l'enchaînement anaphorique est-il difficile entre un village et l'église romane, alors qu'il est acceptable avec des optionnels comme le couteau, les chandeliers, dans les exemples (6) et (7) ? Et, autre problème, pourquoi des enchaînements comme (3), (4) et (5) sont-ils, eux, acceptables?

\section{VALEUR RÉFÉRENTIELLE DE L'ARTICLE DÉFINI ET NATURE DE LA MODIFICATION ADJECTIVALE}

L'explication que je tenterai d'apporter à ces différents problèmes repose principalement sur la valeur d'unicité existentielle que présuppose l'article défini. Cette explication ne se dispensera pas complètement d'un recours aux stéréotypes - autrement dit, les stéréotypes auront encore leur mot à dire dans l'affaire; on verra en particulier que les enchainements anaphoriques sont malheureux dès lors que les informations stéréotypiques s'opposent au calcul de l'unicité.

Comme le souligne Kleiber $(2001,77)$, « il y a [...] un accord assez grand pour voir dans l'article défini un marqueur d'unicité existentielle, qui présente l'entité dénotée comme étant la seule entité à vérifier le contenu descriptif $\mathrm{N}$ ou $\mathrm{N}+$ Modificateur ${ }^{7}$. En AA, J'ensemble dans lequel se vérifie l'unicité est l'élément inclusif constitué par l'antécédent. Ainsi dans les exemples (1a) et (2a), l'église et le plafond sont à chaque fois présentés comme étant les seules entités des catégories "église » et "plafond " à l'intérieur des ensembles un village et une pièce - unicité qui, on peut le préciser, ne contrevient en rien aux informations stéréotypiques associées à un village et à une pièce (un village n'a généralement qu'une église et une pièce qu'un plafond).

\subsection{Des effets contrastifs appropriés ou non}

Comme corollaire à cette valeur d'unicité, le défini marque ce que C. Blanche-Benveniste et A. Chervel (1966) appellent une opposition notionnelle, une saisie d'un signifié en contraste avec d'autres signifiés, ou encore ce que F. Corblin (1983, 1987) exprime en termes de " contraste externe de domaine à domaines " : le défini extrait un Ni parmi des éléments qui n'appartiemnent pas à la classe des $\mathrm{Ni}$. Le $\mathrm{Ni}$ est présenté comme le seul à vérifier I'appartenance à la catégorie Ni dans un ensemble donné et il est ainsi le Ni par opposition à tout ce qui n'appartient pas à la classe des $\mathrm{Ni}$.

7. Pour une vraie présentation de la question, voir Kleiber (1992a et 2001). 
Dans une séquence anaphorique comme (1a), le SN défini l'église extrait une " partie » unique parmi l'ensemble des « parties » du village. L'unicité est vérifiée dans l'ensemble un village et la valeur de contraste externe associée à l'article défini trouve sa justification par rapport aux autres "parties " constitutives de cet ensemble. En revanche, dans une séquence comme (1b), l'occurrence du défini ne s'explique que si l'église romane peut s'opposer à une autre église, c'est-à-dire que l'ajout d'un modificateur restrictif ne permet plus ici l'opposition aux autres "parties " constitutives du village, mais suppose un contraste externe avec un autre (voire d'autres) élément(s) de l'ensemble des églises du village. En restreignant l'extension du nom, le modificateur restrictif romane moditie l'ensemble sur lequel s'opère l'extraction, le lieu du calcul de l'unicité est déplacé ${ }^{8}$ : il ne s'agit plus de la seule entité "église " de l'ensemble un village, mais de la seule entité "église romane " parmi l'ensemble des églises d'un village, lesquelles ne seraient pas toutes de type roman. Or, et c'est là que les stéréotypes retont surface, cette opposition, cette valeur distinctive est a priori improbable, puisqu'un village stéréotypique ne possède qu'une église. Ce qui heurte l'acceptabilité d'un enchaînement comme (1b), c'est finalement l'incongruité de l'effet contrastif par rapport à la représentation stéréotypique de l'ensemble.

Ce type d'effet contrastif indésirable, lié à la cooccurrence d'un article défini et d'un modificateur restrictif, se rencontre dans d'autres configurations anaphoriques. A. Theissen (2001a et b) l'a relevé dans des exemples d'anaphores fidèles tels que (16) et (17):

(16) "Un tracteur rouge ronronnait dans le champ. Le voisin, Nyberg, travaillait sur les terres de Ronkaila. Il épandait apparemment un produit contre les maniaises herbes : il y avait derrière le tracteur (? le tracteur rouge) un grand réservoir en plastique, d'où jaillissait un brouillard de liquide empoisomé.. "(A. Paasilina, Le fils du dieu de l'Orage, cité par Theissen 2001a, 51)

(17) "Le poète se passa la main sur le visage, comme un homme qui vient de se réveiller, et il vit que le soir tombait sur l'étnng du patriarche. L'eau était noire, ct, déjà, une barque légère y glissait. On entendait le clapotis des rames et les rires d'une citoyenne installée dans la barque (? la barque légère). " (M. Boulgakov, Le maître et Marguerite, cité par Theissen 2001a, 51)

exemples auxquels on peut ajouter le fameux exemple de M. Gross $(1977,118)$ :

(18) Un étudiant est entré dans le burean, le gros étudiant a demandé l'heure. ${ }^{9}$

Comme Theissen $(2001 a, 52)$ le remarque fort bien, dans ces exemples-là :

"l'adjectif intervient, à tort, dans le calcul d'unicité en modifiant le lieu de ce calcul. L'unicité ne paraît plus être calculée au sein de la catégorie $\mathrm{N}$ - et donc à partir du nom seul comme c'est le cas pour le $\mathrm{N}$ - mais s'établit à partir d'une catégorie, en quelque sorte, subordonnée à celle dénommée par $\mathrm{N}$, qui est $N / \mathrm{Mod}$. "

8. Cf. infra, Theissen (2001a et b).

9. L'exemple de Gross, un peu différent de ceux donnés par Theissen, puisque la spécification adjectivale y apporte une information nouvelle, absente du contexte antécédent, est destiné à montrer que l'ajout d'un modificateur restrictif - contrairement à celui d'un modificateur explicatif (non restrictif), of. l'exemple (19) - exchut toute interprétation coréférentielle entre les deux $\mathrm{SN}$ ayant pour tête le nom étudiant. 
La répétition adjectivale dans l'anaphorique (cf. exemples (16) et (17)) produit ainsi un effet contrastif comparable à celui de notre église romane : dans les deux cas - anaphore fidèle et AA - le calcul d'unicité s'opère sur une catégorie plus étroite (tracteur rouge, barque légère, église ronane) et suppose une opposition contextuelle possible à d'autres éléments de la catégorie plus large des tracteurs, des barques ou des églises.

On ajoutera que, dès que le modificateur ne restreint plus l'extension du nom, c'est-à-dire dès qu'il s'agit d'un modificateur purement descriptif, ces configurations anaphoriques ne posent plus ce type de problème ${ }^{10}$ :

(19) Un étudiant est entré dans le bureau, l'étudiant, affolé, a demandé l'heure. (M. Gross 1977, 118)

(20) J'ai jeté un coup d'ail dans une pièce. Le plafond, jaune, était très haut.

Le problème de l'église romane en (1b) ou du plafond jaune en (2b) ne réside donc pas seulement dans la nature non conventionnelle de la caractérisation adjectivale, mais aussi et surtout dans la quantité plurielle que présupposent les expressions définies l'église romane, le plafond jaune. C'est-à-dire que la faible acceptabilité des séquences (1b) et (2b) est comparable à celle des deux séquences suivantes:

(21) ? Nous entrâmes dans un village. Les églises étaient situées sur une hauteur. (Kleiber 1993a, 362 et 1993b, 58)

(22) ?J'ai jeté un coup d'ail dans une pièce. Les plafonds étaient très hauts.

Toutes ces séquences contreviennent, à leur manière, à la quantité de "parties " inscrite dans le savoir conventionnel ${ }^{11}$. Et, lorsqu'on a pour antécédent un " tout " dont la représentation stéréotypique n'exclut pas l'existence de plusieurs "parties " d'un même type, l'ajout d'un modificateur restrictif ne paraît plus poser de difficultés :

(23) a. Au loin nous aperşûmes une ville. Les églises gothiques s'élevaient bien plus haut que les églises romanes.

b. Hier, nous avons visité une ville. Nous avons été très impressionnés par les églises gothiques / par la beauté des églises gothiques.

(5) «Nous habitions un grand appartement plein de choses étranges. (...) Tout l'appartement était rempli de curiosités naturelles. Seul, le petit salon n'avait été enviahi ni par la zoologie, ni par la minéralogie, ni par l'ethnographie, ni par la tératologie (...) » (A. France, Le livre de mon ami)

Dans l'exemple (5), la détermination adjectivale petit circonscrit un sousensemble de salons et le $\mathrm{SN}$ défini le petit salon marque une opposition à au

10. On n'appliquera pas ce type de modification à l'exemple-phare du village et de l'église romane, dans la mesure où l'adjectif romane a une valeur intrinsèquement distinctive qui s'accommode mal et de la fonction d'apposition (? L'église, romene, était siluée sur une hauteur) et de la fonction attribut (? Cette église est romane). On ne l'appliquera pas non plus aux deux exemıles de Theissen (2001a) pour d'autres raisons: lorsque les adjectifs rouge, légère sont apposés aux SN anaphoriques le tracteur, la barque, les effets contrastifs indésirables disparaissent, mais les séquences ne deviennent pas acceptables pour autant ; la répétition d'adjectifs déjà présents dans les $\mathrm{SN}$ antécédents semble redondante, peu pertinente d'un point de vue informatif.

11. Sur l'importance de la quantité pour l'AA, voir Kleibler (1993a et b, 2001). 
moins un autre salon (vraisemblablement un grand salon) ${ }^{12}$. On suppose alors que l'appartement (le grand appartement) dont il est question ici comprend plusieurs salons. Et même si cette quantité plurielle caractérise assez mal notre représentation actuelle d'un appartement (caractérise-t-elle mieux celle qu'on pouvait en avoir à la fin du XIXe siècle ?), elle reste possible et ne rend pas le tout atypique.

\subsection{Contextes réfractaires}

Toutes les configurations anaphoriques ne seront pas pour autant possibles. Une séquence comme (24), par exemple, reste difficile, bien qu'elle ne déroge pas à la quantité stéréotypique :

(24) ? Hier, nous avons visité une ville, mais nous n'avons pas trouvé les églises gothiques.

Mais ce type de difficultés dépasse le cadre des AA avec modifications adjectivales pour s'appliquer à toutes les AA avec composants possibles (ou optionnels). Ainsi, les enchaînements suivants sont, eux aussi, difficilement recevables :

(25) a. ? Max entra dans une pièce, mais ne trouva pas les chandeliers.

b. ? Jean a été assassiné hier. Le couteau n'a pas été retrouvé.

Dans tous ces exemples, la saturation référentielle des SN définis (les églises gothiques, les chandeliers, le couteau) ne peut pas être assurée par la seule relation associative avec une ville, une pièce, a été assassiné. Elle doit se faire par d'autres moyens. L'indétermination référentielle reste entière (quelles églises gothiques? quels chandeliers ? quel couteau ?) dans les contextes réduits de (24) et (25), alors qu'avec des composants typiques, attendus, ces mêmes enchaînements seraient tout à fait acceptables :

a. Max entra dans une chambre, mais ne trouva pas le litlles lits.

b. Jean a été assassiné hier. L'arme n'a pas été retroưuée.

Le statut de ces divers composants (i.e. composants possibles versus composants attendus, typiques) explique aisément ces différences de comportement en AA. L'attente, quand on entre dans une chambre, c'est d'y trouver un ou plusieurs lits. Ne pas en trouver contrarie donc cette attente. (26a) pourrait se gloser par "Max ne trouva pas le lit, élément qu'on trouve habituellement dans une chambre " et la définitude de lit ne pose alors aucun problème ${ }^{13}$. Rien de semblable avec des chandeliers quand on entre dans une pièce ou des églises gothiques quand on visite une ville: ne pas trouver de chandeliers dans une pièce ou d'églises gothiques dans une ville n'a rien de surprenant et ne

12. L'exemple suivant met bien en évidence l'interprétation contrastive associée au SN défini le petit salon :

"Après le dîner, nous passêmes au "petit salon", formule que Renée s'obstine à employer dès que nous avons des visiteurs, bien qu'il n'y en ait qu'un, ni maiment petit, ni waiment grand”. (A. Maupin, Mrybe the Moon, traduction française de F. Rosso, Passage du Marais, 1999, p. 234).

13. On constate ainsi, fait déjà relevé par Corblin $(1987,133)$, que l'AA n'exige pas toujours l'existence du référent de l'anaphorique ( $c f$. aussi les deux exemples suivants empruntés à Corblin : $l l y$ a bien une église dans le village, mais le curé n'a janais été nommé ; On ne peut vendre cette voiture, car le volant a été oublić lors du montage). 
contrevient en rien à notre attente ${ }^{14}$, à moins de savoir au préalable que cette pièce-ci contient, parmi son mobilier, des chandeliers et que cette ville-ci compte parmi son patrimoine, des églises gothiques. Ces composants n'étant pas attendus par la simple mention d'une pièce ou d'une ville, leur définitude exige nécessairement, dans des contextes de ce type, une identification préalable.

\subsection{Contextes favorables}

Le problème des enchaînements (1b) et (2b) ne relève donc pas seulement du caractère contingent de la spécification adjectivale; je précise bien pas seulement, car la nature conventionnelle ou non de la caractérisation adjectivale a évidemment son rôle à jouer. Et, lorsque l'église romane est un composant connu d'un village identifié (comme en (27)), ou un composant probable, attendu, d'un village qui, bien qu'il soit présenté sous la forme d'un SN indéfini, n'est pas totalement indéterminé (comme en (28), où le contexte antécédent construit par M. Charolles circonscrit en fait une sous-classe de villages, ceux de la Bourgogne romane; $c f$. G. Kleiber 1994a et 2001), l'expression définie ne produit plus les mêmes effets contrastifs et l'enchaînement anaphorique redevient possible ${ }^{15}$ :

(27) Nous arrivâmes dans (le village de) Pierrecombes. L'église romane qui se trouve. sur le haut était fermée. (Kleiber 1994b, 162)

(28) Nous nous étions inscrits à un circuit "Connaissance de la Bourgogne romañe". La région était littéralement couverte de petits édifices dont certains remontaient au $\lambda^{\prime \prime}$ siècle. Nous partînes de bon matin car le programme était chargé. Nous arrivâmes dans un village. L'église romane était située sur une hauteur. (...). (Charolles 1994, 79)

\subsection{Modification adjectivale descriptive}

Il reste encore à expliquer pourquoi les séquences (3) et (4) sont acceptables alors que, cette fois - contrairement aux exemples (5) et (23) - la représentation stéréotypique des « touts » exclut radicalement une quantité plurielle de parties (une pièce n'a qu'un plafond, des femmes n'ont qu'un corps, qu'un dos, qu'une nuque...):

(3) "Au dernier crépuscule, aux premières étoiles, ces dames arrivent, avec des révérences adorables. Et bientôt, notre maisonnette est pleine de petites femmes accroupies, dont

\footnotetext{
14. Ce que souligne d'ailleurs la version négative du test en mais utilisé plus haut:

? C'est une pièce, mais il n'y a pas de chandeliers. (Pourquoi y en aurait-il ?)

? C'est une ville, mais il n'y a pas d'églises gothiques. (Pourquoi y en aurait-il ?)

qu'on opposera à :

C'est une chambre, mais il n'y a pas de lit(s).
}

15. À ce propos, on rappellera l'importance, signalée par Kleiber (1993a et b, 1994b et 2001), d'étudier des séquences anaphoriques avec un antécédent indéfini : un SN indéfini présente le N en question comme un membre stéréotypique de la classe des $\mathrm{N}$, alors qu'avec un SN défini (ou même le $\mathrm{SN}$ indéfini de (28), où le contexte apporte déjà d'autres informations que celles fournies par le seul $\mathrm{N}$ village : le village, ici, n'est pas seulement présenté comme un membre de la classe des villages, mais, de façon plus spécifique, comme un membre de la sous-classe des villages de la Bourgogne romane), le référent " apparaît comme un référent commu autrement que par sa seule appartenance à une classe de $\mathrm{N}$ " (Kleiber 1994b, 155). 
les yeux bridés sourient vaguement, on voit luire comme de l'ébène poli tous les beaux chignons aux coques soignées; les corps frêles se perdent dans les plis des vêtements trop larges, qui bâillent tous, comme prêts à tomber, sur les petits dos fuyants, et découvrent les nuques exquises. "(P. Loti, Madame Chrysanthème)

J'ai jeté un coup d'ceil dans une pièce. Le plafond jaune jurait avec les murs rouges.

J'ai suggéré plus haut que tous mes contre-exemples n'avaient peut-être pas la même validité, tous ne présentant sans doute pas un modificateur à valeur restrictive. $\mathrm{Si}$, dans ces deux séquences, la combinaison de l'adjectif et du défini ne produit pas les effets contrastifs indésirables observés dans d'autres séquences, cela tient vraisemblablement à la nature descriptive de l'adjectif. Les modifications adjectivales ne restreignent plus ici l'extension du nom et n'ont ainsi aucune incidence sur le calcul référentiel du SN ; le calcul d'unicité s'opère à partir du nom seul et non à partir d'une catégorie plus restreinte $N+$ adj. Il faudrait toutefois préciser ce qui, dans ces deux exemples, favorise l'interprétation descriptive des modifications adjectivales et, en particulier, pourquoi jaune, qui est épithète dans les deux cas, serait descriptif en (4) et restrictif en (2b) :

(2) b. ? J'ai jeté un coup d'aril dans une pièce. Le plafond jaune était très haut. (Kleiber 1993b, 57)

Dans nos deux exemples, c'est probablement la pluralité de parties évoquées qui contribue à l'interprétation non restrictive des épithètes : la mise en contraste explicite de diverses parties favorise apparemment l'interprétation descriptive des modifications adjectivales (en (4), par exemple, le plafond janne s'oppose explicitement aux murs rouges, et non à un autre plafond). Ces modifications adjectivales représentent ainsi une série de qualifications accessoires dont la pertinence apparaît clairement dans le cadre d'une énumération descriptive (comme c'est le cas dans l'extrait de Loti) ou d'une mise en perspective des couleurs de diverses parties (comme dans l'exemple (4)). On peut estimer que les énumérations de parties constitueront toujours un cadre relativement favorable à la modification adjectivale en AA, à condition bien entendu que l'adjectif autorise une interprétation descriptive ${ }^{16}$. Les exemples suivants tendent à le confirmer :

(29) «M. Lebigre tennit un fort bel établissement, d'un luxe tout moderne. Placé à l'encoignure droite de la rue Rambutean, flanqué de quatre petits pins de Norvège dans des caisses peintes en vert, il faisait un digne pendant à la grande charcuterie des Quenu-Gradelle. Les glaces claires laissaient voir la salle, ornée de guirlandes de feuillages, de pampres et de grappes, sur un fond vert tendre. Le dallage était noir et blanc, à grands carreaux. Au fond, le trou béant de la cave s'ouvrait sous l'escalier tournant, à draperie rouge, qui menait au billard du premier étnge. Mais le comptoir surtout, à droite, était très riche, avec son large reflet d'argent poli. (...) " (Zola, Le ventre de Paris, Folio/Gallimard, 2002, p. 168).

16. Ce qui n'est pas le cas des adjectifs relationnels ou encore des adjectifs que G. Gross $(1996,51)$ appelle typologisants (e.g. accent aigu/grave/circonflexe, enseignement primaire/secondaire/supírieur), qui ont une valeur distinctive, sous-catégorisante par nature. 
(30) "Petit et gros, brusque et leste comme un singe quoique d'un caractère calme, Michu avait une face blanche, injectée de sang, ramassée comme celle d'un Calnouque et à laquelle des cheveux rouges, crépus donnaient une expression sinistre. (...) Cette figure socratique à nez camus était couronnée par un très beau front, mais si bombé qu'il paraissait être èn surplomb sur le visage. Les oreilles bien détacliées possédaient une sorte de mobilité comme celles des bêtes sauvages, toujours sur le qui-vive. La bouche, entr'ouverte par une habitude assez ordinaire chez les campagnards, laissait woir des dents forte's ef blanches comme des amandes, mais mal rangées. (...)" (Balzac, Une ténébreuse affaire, Folio/Gallimard, 1973, p. 24-25)

\section{POUR CONCLURE}

On a pu constater, grâce à des séquences comme (5) et (23), que l'ajout d'un modificateur restrictif non stéréotypique dans le SN anaphorique n'entravait pas toujours le bon fonctionnement de l'AA. L'occurrence d'un tel modificateur, à partir du moment où celui-ci ne rend pas la "partie " atypique ${ }^{17}$, est tributaire de la nature unique ou plurielle de la " partie ", en d'autres termes de la quantité stéréotypique de " parties " : avec une partie unique, la modification restrictive produit nécessairement des effets contrastifs inappropriés; avec une partie plurielle, les effets contrastifs produits par le modificateur restrictif sont légitimes puisqu'ils ne contreviennent plus à la quantité de parties inscrite dans le savoir conventionnel.

La différence observée entre le comportement des modifications adjectivales et celui des composants optionnels en AA, malgré une parenté certaine, s'explique finalement par le mode de calcul référentiel lié à la cooccurrence d'un article défini et d'un modificateur restrictif : en effet, l'ajout d'un modificateur restrictif déplace le calcul d'unicité d'une catégorie $\mathrm{N}$ à une catégorie $\mathrm{N}$ + adj, ce qui produit ipso facto des effets contrastifs dont le caractère approprié ou non dépend de la quantité stéréotypique de « parties».

La valeur référentielle de l'article défini et le caractère restrictif de l'adjectif figurent donc au centre de la question de la modification adjectivale en AA. Reste à déterminer, de façon nettement plus rigoureuse que je ne l'ai fait ici, ce qui autorise une interprétation non restrictive de l'adjectif dans des exemples comme (3) ou (4).

17. On opposera à ce propos une modification adjectivale comme romane dans le couple village/ église romane, à la modification adjectivale grand qu'on trouve dans un autre couple célèbre de Kleiber, village/grand magasin. Cette dernière modification, contrairement à la première, ne définit plus une spécificité possible, mais une spécificité atypique qui l'exclut radicalement de suites anaphoriques comme :

? Nous entrâmes dans un village. Le grand magasin était fermé. (Kleiber 1992b, 189)

Grand circonscrit ici un type de magasin de village inattendu et n'autorise ainsi aucun rapprochement avec les optionnels ou parties possibles de Kleiber. 


\section{Bibliographie}

Blanche-Benveniste, Claire et Chervel, André (1966), "Recherches sur le syntagme substantif ", Cahiers de lexicologie, IX, 2, p. 3-37.

BRown, Gillian et YULE, George (1983), Discourse analysis, Cambridge, Cambridge University Press.

CHAROLles, Michel (1994), "Anaphore associative, stéréotype et discours ", in L'anaphore associative, Recherches linguistiques, XIX, C. Schnedecker, M. Charolles, G. Kleiber \& J. David (éds), Paris, Klincksieck, p. 67-92.

CLARK, Herbert H. (1977), "Bridging ", in Thinking. P.N. Johnson-Laird \& P.C. Wason (eds), Cambridge, Cambridge University Press, p. 411-420.

Corblin, Francis (1983), "Défini et démonstratif dans la reprise inmmédiate ", Le Français moderne, 51, 2, p. 118-134.

CoRblin, Francis (1987), Indéfini, défini et démonstratif, Genève, Droz.

CRuse, D. Alan (1986), Lexical semantics, Cambridge, Cambridge Textbooks in Linguistics, Canbridge University Press.

Gross, Gaston (1996), Les expressions figées en français, Gap-Paris, Ophrys.

GROSS, Maurice (1977), Grammaire transformationnelle du français. Syntaxe du nom, Paris, Larousse.

KLEIBER, Georges (1992a), "Article défini, unicité et pertinence ", Revue Romane, 27, 1, p. 61-89.

KLEIBER, Georges (1992b), "Anaphore associative et inférences ", in Lexique et inférence(s), Recherches linguistiques, XVIII (VII Colloque International de Linguistique. 1416 novembre 1991, Metz), J.-E. Tyvaert (éd.), Paris, Klincksieck, p. 175-201.

KLEIBER, Georges (1993a), "L'anaphore associative roule-t-elle ou non sur des stéréotypes? "in Lieux communs - topoï, stéréotypes, clichés, C. Plantin (éd.), Paris, Kimé, p. 355-371.

KLEIBER, Georges (1993b), "Anaphore associative, pontage, stéréotypie ", Linguisticae Investigationes, XVII, 1, p. 35-82.

KLEIBER, Georges (1994a), Discours et stéréotypie : le contexte peut-il remettre d'aplomb une anaphore associative mal formée? L'Anaphore associative, Recherches linguistiques, XIX, C. Schnedecker, M. Charolles, G. Kleiber \& J. David (éds). Paris : Klincksieck, p. 93-116.

KLEIBER, Georges (1994b), "Anaphore associative, antécédent et définitude ", in L'anaphore associative, Recherches linguistiques, XIX, C. Schnedecker, M. Charolles, G. Kleiber \& J. David (éds), Paris, Klincksieck, p. 153-173.

KLEIBER, Georges (1995), "Anaphore associative, thèse lexico-stérotypique : oui, mais... ", Cahiers de praxématique, 24, p. 69-85.

KLEIBER, Georges (2001), L'anaphore associative, Paris, PUF.

THEISSEN, Anne (2001a), "La concurrence entre un SN défini fidèle et un SN défini totalement fidèle ou Comment expliquer le maintien ou non de l'adjectif dans un SN défini anaphorique basique? ", in Anaphores pronominales et nominales. Études pragma-sémantiques, W. De Mulder, C. Vet \& C. Vetters (éds), Amsterdam-New York. Rodopi, p. 43-67.

THEISSEN, Anne (2001b), "Quelques observations sur l'apparition d'un adjectif dans un SN défini de reprise ", Par monts et par vaux. Itinéraires linguistiques et grammaticaux (Mélanges offerts à Martin Riegel). C. Buridant, G. Kleiber \& J.-C. Pellat (éds). LouvainParis, Peeters, p. 407-416. 